\title{
Synthesis and properties of latent pigment nano- and micro-encapsulated in high-durability inorganic capsules
}

\author{
Tomoji Ohishi* and Takaki Kawamura \\ Department of Applied Chemistry, Faculty of Engineering, Shibaura Institute of Technology, 3-7-5 Toyosu, Koto-ku, Tokyo 135-8548, Japan
}

\begin{abstract}
Preparation and properties of latent pigment nano- and micro-encapsulated in inorganic capsule have been investigated. Sol-gel derived $\mathrm{C}_{6} \mathrm{H}_{5}-\mathrm{SiO}_{2}$ sphere capsules containing organic pigment were prepared using solvent-soluble latent pigment. By controlling the sol-gel reaction of $\mathrm{C}_{6} \mathrm{H}_{5}-\mathrm{Si}_{2}\left(\mathrm{OC}_{2} \mathrm{H}_{5}\right)_{3}$ as starting material by acidic catalyst, followed by basic catalyst, it was possible to prepare spherical inorganic capsules containing organic pigment with micro- and nano-size. Multi-colored inorganic sphere capsules ware prepared by using the three primary colors of latent pigments (Qn-BOC, Indigo-BOC, and PY-BOC). Colored microcapsules (MCs) with excellent heat resistance, light resistance, and coloring property were obtained. Inorganic micro- and nano-capsules of Qn-BOC showed strong fluorescenceemission characteristics.
\end{abstract}

\section{Introduction}

Microcapsules (MCs) are rapidly being developed as materials for applications such as information recording, cosmetics, coatings, and food stuffs. In particular, as for microencapsulation of functional organic dyes, it is expected to be applied to, for example, display materials, information-recording materials, microsphere lasers, biomaterials such as drug delivery system and in-vivo fluorescent dye materials, and it is a field in which great development is expected in the future [1-13]. Conventionally, MCs of functional organic dyes have a structure in which an organic dye forms a core that is encased by an organic-polymer shell. However, due to the low durability of the dye, such MCs have been insufficient in terms of performance. Although organic pigments with good durability have been used, they are not soluble in a solvent, so it is difficult to make them into microparticles and microencapsulate them, and good performance has not been achieved [8]. In general, mechanical pulverization by a bead mill or the like is mainly used for micronizing an organic pigment $[14,15]$. A point of issue with that processing, however, is that the resulting particle shape is non-uniform, and spherical MCs cannot be obtained. Furthermore, when organic pigments are used, organic polymers are used for the shell structure; consequently, heat resistance, light resistance and mechanical durability (such as hardness) are low.

The present study aimed to microencapsulate high-durability and high-performance functional organic pigment in inorganic polymer. In particular, organic-solvent-soluble latent pigment was microencapsulated in inorganic MCs formed from inorganic polymer synthesized by the sol-gel method. In that manner, inorganic MCs containing organic pigment were synthesized, and the properties of the MCs were investigated. Starting as an organic pigment, a latent pigment is made soluble in various solvents by introducing an organic substituent into an insoluble organic pigment [16]. A latent pigment has the property that it can be returned to the original pigment by heat treatment. For that reason, it can be easily introduced into a capsule because it has solubility like a dye being introduced into a film, and after the pigment is transformed by heat treatment, it manifests high durability. Organic-pigment-containing inorganic MCs (MCs) were synthesized by combining the latent pigment with an inorganic polymer synthesized by the sol-gel method. As for the inorganic polymer (synthesized by the sol-gel method), phenyltriethoxysilane was used as the starting material, and MCs with spherical shape were made by coating an organic pigment with an inorganic polymer $\left(\mathrm{C}_{6} \mathrm{H}_{5}-\mathrm{SiO}_{2}: \mathrm{Ph}-\mathrm{SiO}_{2}\right)$, in which a phenyl group is chemically bonded to a siloxane skeleton. In this manner, organic-pigment-containing inorganic MCs with higher durability than MCs formed with organic resin could be developed. In addition, it was possible to make MCs of various colors by using latent pigments of the three primary colors as coloring material.

\section{Experiment}

\section{Synthesis of latent pigment}

The latent pigment was synthesized with three primary-color pigments as coloring materials, namely, magenta-colored quinacridone (Pigment Violet 19: Dainichiseikakougyo Co. Ltd.), indigo (for cyan) (Tokyo Chemical Inc.), and a diazo yellow (for yellow) (Pigment Yellow 93: Dainichiseikakougyo Co., Ltd.), by a previously reported method [17].

\section{Synthesis of inorganic micro- and nano-capsules}

$2.40 \mathrm{~g}(9.98 \mathrm{mmol})$ of phenyltriethoxysilane (PTES; Shin-Etsu Chemical Co., Ltd.) was mixed with $3.00 \mathrm{~g}$ of $\mathrm{HCl}$ (adjusted to $\mathrm{pH} 3$ ), and the mixture was stirred at room temperature for 24 hours. As the

Correspondence to: Tomoji Ohishi, Department of Applied Chemistry, Faculty of Engineering, Shibaura Institute of Technology, 3-7-5 Toyosu, Koto-ku, Tokyo 135-8548, Japan, Tel: +81-3-5859-8154; E-mail: tooishi@sic.shibaura-it.ac.jp

Key words: Micro- and Nano-capsules, Latent pigment, Inorganic capsule, Sol-gel method, High durability, Multi-colored capsules

Received: November 04, 2017; Accepted: November 21, 2017; Published: November 24, 2017 
polymerization reaction progressed, the solution became an opaque emulsion. When the solution was allowed to stand for 72 hours, it became separated into two layers: one layer of $\mathrm{C}_{6} \mathrm{H}-\mathrm{SiO}_{2}$ polymer and one layer of hydrochloric-acid aqueous solution. The upper layer (hydrochloric-acid aqueous solution) was removed, and $\mathrm{a} \mathrm{SiO}_{2}$ polymer sol having a phenyl $(\mathrm{Ph})$ group was obtained by a sol-gel reaction. The $\mathrm{Ph}-\mathrm{SiO}_{2}$ polymer sol was dropped into $\mathrm{NH}_{4} \mathrm{OH}$ solution (adjusted to a constant amount of $13 \mathrm{~mol} / \mathrm{L}$ ) by using a micropipette and stirred for 30 minutes, after which MCs settled out. The produced MCs were separated and dried at $100^{\circ} \mathrm{C}$ for 30 minutes. Then, 5- to 80 -wt\% solutions in which the $\mathrm{Ph}-\mathrm{SiO}_{2}$ polymer sol was dissolved in 1,4-dioxane were prepared, and those solutions were used to prepare MCs in the same manner as described above.

\section{Synthesis of latent-pigment-containing inorganic micro- and nano-capsules}

Latent pigment was added to and mixed with a solution prepared by dissolving the $\mathrm{Ph}-\mathrm{SiO}_{2}$ polymer sol (prepared as described in Section 2.2) in 1,4-dioxane to give a homogeneous solution. When that solution was added dropwise to an ammonia solution $(13 \mathrm{~mol} / \mathrm{L})$, MCs containing the latent pigment were generated. The MCs were taken out and dried at $100^{\circ} \mathrm{C}$ for 30 minutes. They were then heat-treated at $160^{\circ} \mathrm{C}$ for 30 minutes to convert the latent pigment into an organic pigment encapsulated in the inorganic polymer MCs.

\section{Evaluation}

To determine the molecular structure of the prepared $\mathrm{Ph}-\mathrm{SiO}_{2} \mathrm{MCs}$, an infrared absorption spectrum was measured (with a Shimadu FTIR8400 ) by the KBr method. Differential thermogravimetric analysis was also performed with a Shimadu TG-50. The surface morphorogy of the MCs was observed by optical microscope and scanning electron microscope (SEM; KEYENCE VE-8800). A light-resistance test (an accelerated ultraviolet exposure test) was then performed. In detail, MCs were spin-coated on a quartz substrate, irradiated with ultraviolet light (wavelength: $365 \mathrm{~nm}$; intensity: $614 \mu \mathrm{W} / \mathrm{cm}^{2}$ ) for a certain time, and the change in the light-resistance property was tracked by using a visible-ultraviolet transmission spectrum (Shimadu UV-2450).

\section{Result and discussion}

\section{Preparation and properties of inorganic MCs}

The synthetic route for the MCs is shown in Figure 1. To prepare inorganic MCs with high durability, PTES $\left(\mathrm{C}_{6} \mathrm{H}_{5} \mathrm{Si}\left(\mathrm{OC}_{2} \mathrm{H}_{5}\right)_{3}\right)$ is used as a starting material, and an inorganic-polymer precursor (in which a phenyl group is bonded to a siloxane skeleton) is synthesized by the sol-gel method with an acidic catalyst using $\mathrm{HCl}$. Then, MCs are formed by developing this precursor in $\mathrm{NH}_{4} \mathrm{OH}$ (which is a basic catalyst). After the inorganic polymer is generated as straight chains by using acidic catalyst, three-dimensional condensation is promoted by an accelerating reaction with an alkali catalyst, and spherical MCs are subsequently obtained. By introducing a phenyl group into the inorganic polymer network, compatibility with a latent pigment having an aromatic ring is improved, incorporating the pigment into the inorganic polymer becomes easy, and the coloring property of the pigment is improved. By accelerating the two-step sol-gel reaction with the acidic and basic catalysts, a spherical inorganic capsule was successfully synthesized.

The procedure for preparing the MCs is shown schematically in Figure 2. When PTES was added in a hydrochloric-acid aqueous solution of $\mathrm{pH} 3$ and stirred, they did not mix and formed an emulsion state. When this emulsion-forming reaction was continued for a certain period of time in this state, a polymerization reaction (by the sol-gel reaction of PTES) proceeded, and a PTES sol was formed. After standing for 72 hours, the PTES sol and the $\mathrm{HCl}$ aqueous solution phase separated; accordingly, after this sol solution was extracted and added in aqueous $\mathrm{NH}_{4} \mathrm{OH}$, spherical MCs were formed. In the case that a latent pigment was included (as described later), a solution of latent pigment and PTES sol dissolved in 1,4-dioxane was added in the $\mathrm{NH}_{4} \mathrm{OH}$. In this way, MCs containing a latent pigment were formed, and by heat-treating the MCs to convert the latent pigment into an organic pigment, and organic-pigment-containing MCs were obtained.

Optical-microscope and SEM images of the prepared inorganic MCs (heat-treated at $160^{\circ} \mathrm{C}$ ) that do not contain the latent pigment are shown in Figure 3. As shown in Figures 3(a) and 3(b), spherical MCs with smooth surface and sizes in the range of 30 to $100 \mu \mathrm{m}$ were obtained. An observation image of the internal structure of an inorganic microcapsule with a diameter of $300 \mu \mathrm{m}$ taken by SEM (i.e., the fracture surface of an inorganic microcapsule) is shown in Figure 3(c). It is clear from the image that the capsule is composed of a core-shell structure in which the inside is hollow. And the latent pigment is mainly contained in this hollow interior. The thickness of the inorganic coating on the hollow exterior shell depended on the size of the inorganic capsule, and when the capsule diameter was large, the thickness of the coating also tended to be large.

\section{Infrared-absorption spectrum and thermogravimetric analysis of inorganic MCs}

Infrared spectra of the fabricated MCs (PTES-MCs) and PTES starting material are compared in Figure 4. In the case of the PTESMCs, absorption due to the $-\mathrm{CH}$ stretching vibration of - OEt $\left(\mathrm{C}_{2} \mathrm{H}_{5}\right)$ from $2900 \mathrm{~cm}^{-1}$ to near $2800 \mathrm{~cm}^{-1}$ seen in PTES disappeared, and wide absorption due to Si-O-Si vibration was observed at 1000 to $1200 \mathrm{~cm}$ ${ }^{1}$. This finding suggests that the condensation reaction proceeded by the sol-gel reaction, and an inorganic polymer was formed. Moreover, absorption due to $\mathrm{CH}$ vibration of the phenyl group was observed near $3100 \mathrm{~cm}^{-1}$ and absorptions of $\mathrm{C}=\mathrm{C}$ and $\mathrm{Si}$ - $\mathrm{Ph}$ bonds of the phenyl group were observed near $1600 \mathrm{~cm}^{-1}$ and around $1430 \mathrm{~cm}^{-1}$, respectively, indicating the MCs have a molecular structure in which a phenyl group is bonded to an inorganic skeleton of $\mathrm{SiO}_{2}$. In addition, absorption due to $\mathrm{Si}-\mathrm{OH}$ was observed at $3660 \mathrm{~cm}^{-1}$, suggesting that an $-\mathrm{OH}$ group was formed in part of the inorganic skeleton of $\mathrm{SiO}_{2}$.

The results of thermogravimetric analysis (TG) of PTES-MCs and polystyrene (PS) (which is an organic polymer) are compared in Figure 5. In the case of PTES-MCs, although a slight weight loss was observed from around $400^{\circ} \mathrm{C}$, a significant weight loss was confirmed from $500^{\circ} \mathrm{C}$ to $650^{\circ} \mathrm{C}$ (namely, about 56\%). That weight-loss percentage roughly agrees with the value (i.e., $52.6 \%$ ) given when the phenyl group in $\mathrm{C}_{6} \mathrm{H}_{5}-\mathrm{SiO}_{2}$ burns off, resulting in weight loss. This result suggests that the PTES-MC has a structure in which a phenyl group directly bonds to a siloxane skeleton. Above $650^{\circ} \mathrm{C}$, the phenyl group was completely combusted, and only the $\mathrm{SiO}_{2}$ skeleton remained. On the contrary, in the case of PS, weight loss began from $200^{\circ} \mathrm{C}$, and a large weight loss was observed at 300 to $400^{\circ} \mathrm{C}$. At $400^{\circ} \mathrm{C}$, combustion of PS was complete; that is, weight loss was $100 \%$. This result demonstrates that the prepared MCs have very high heat resistance compared with the organic polymer used for conventional microencapsulation, i.e., PS.

\section{Particle-size control of MCs and factors affecting particle size}

The size of the microcapsule particles depended on the concentration of the inorganic-polymer precursor used during preparation of the 


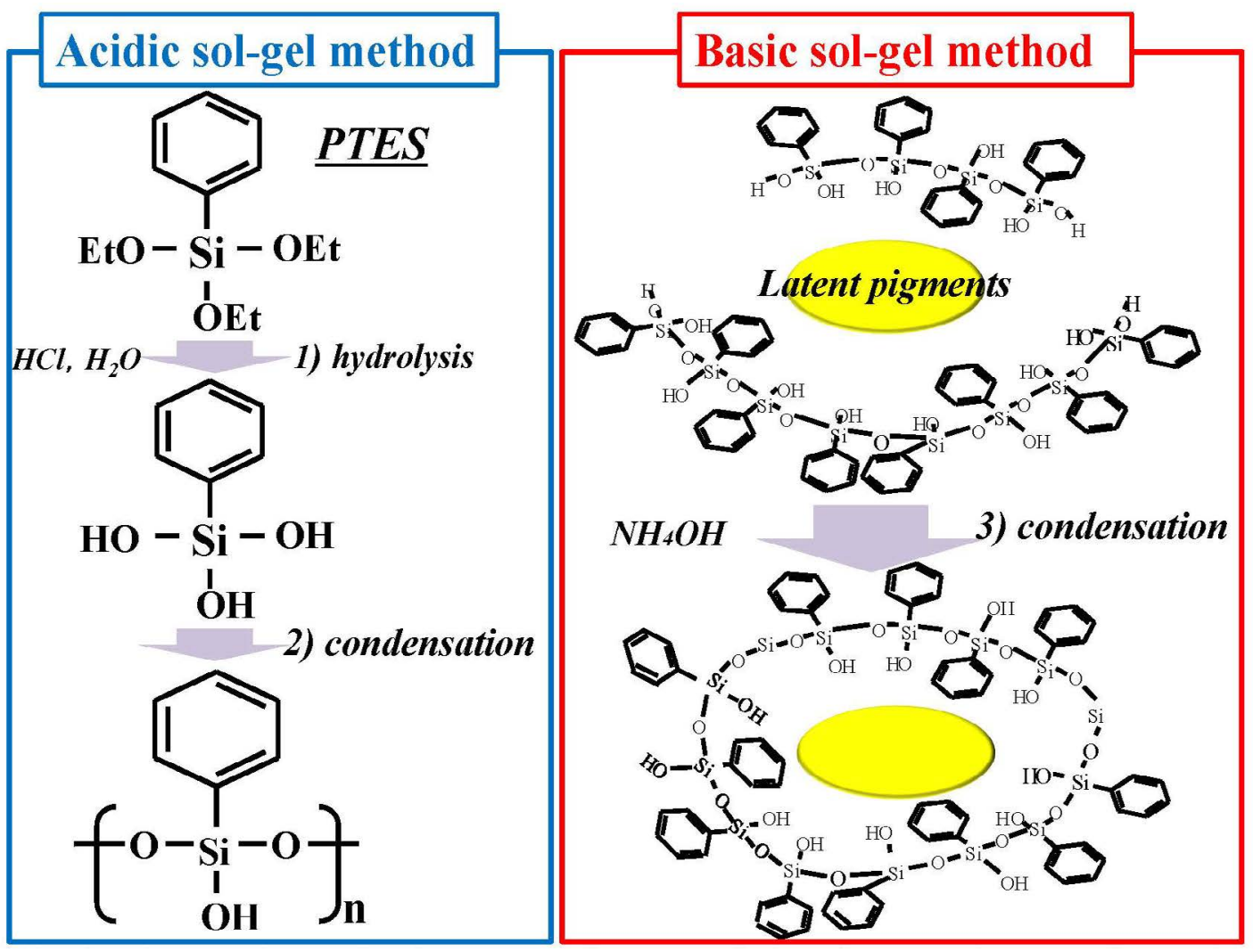

Figure 1. Formation of microcapsule containing latent pigment by using acid and basic sol-gel method

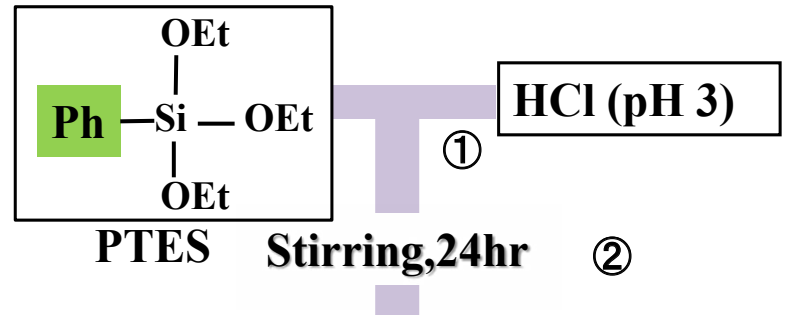

Standing,72hr (3)

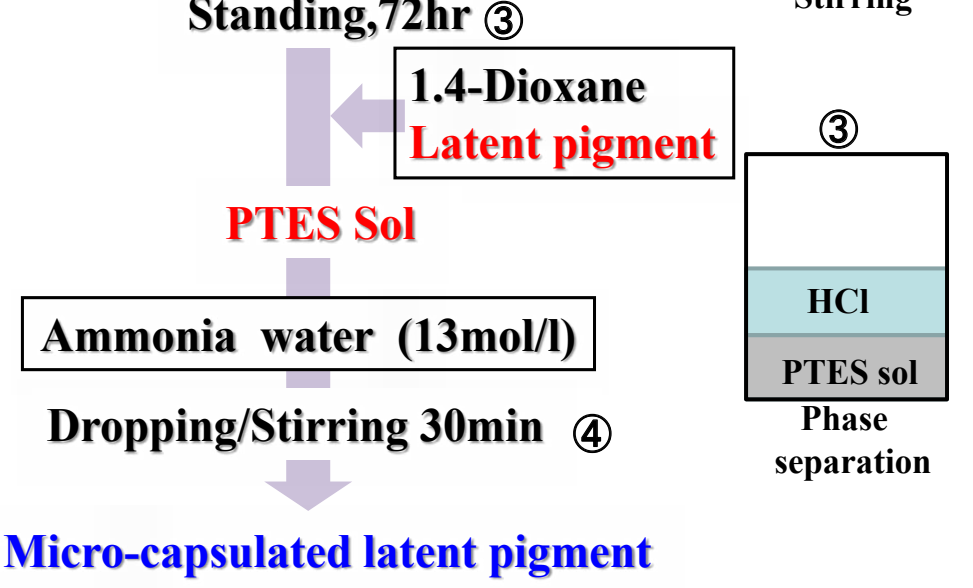

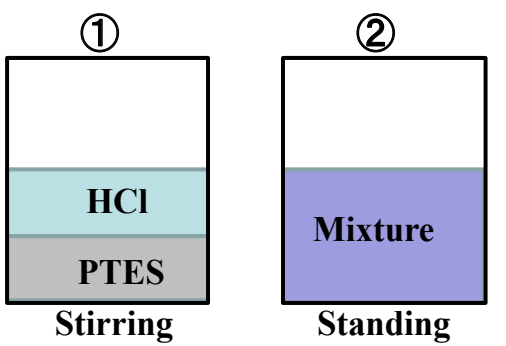

(4)

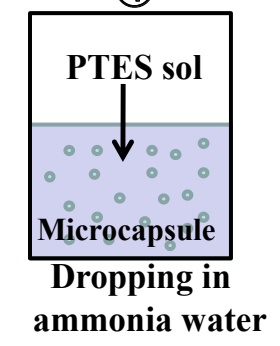

Figure 2. Preparation procedure of microcapsule (MC) 


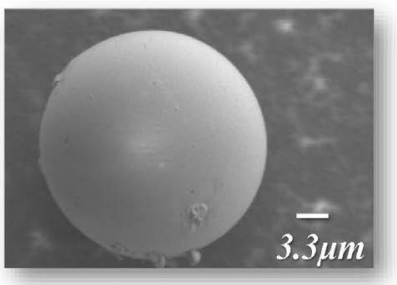

(b) SEM image

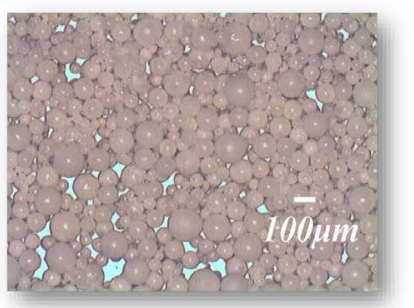

(a) Optical microscope image

Figure 3. Optical microscope and SEM images of microcapsule (MC)

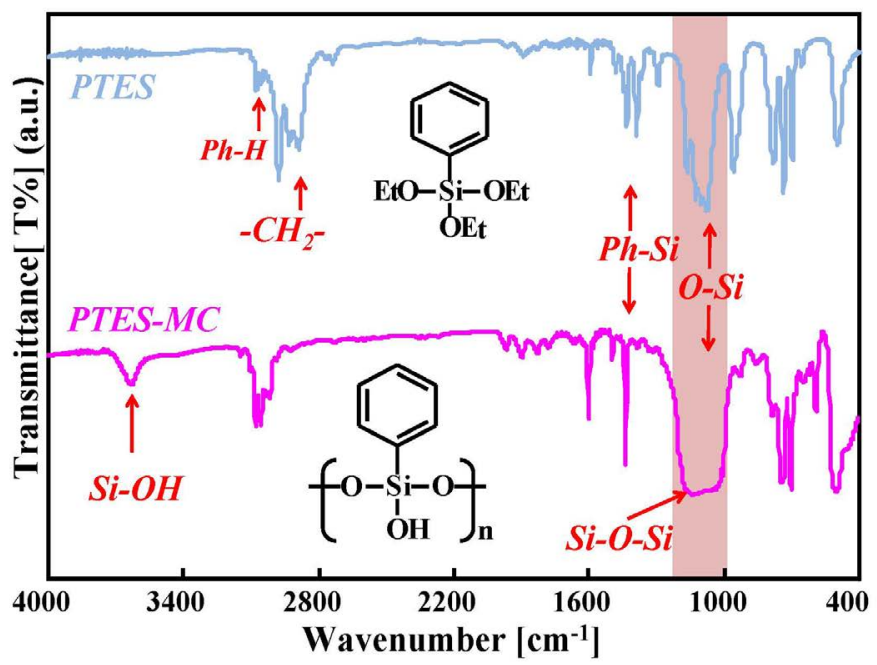

Figure 4. Infrared spectra of PTES-MC and PTES

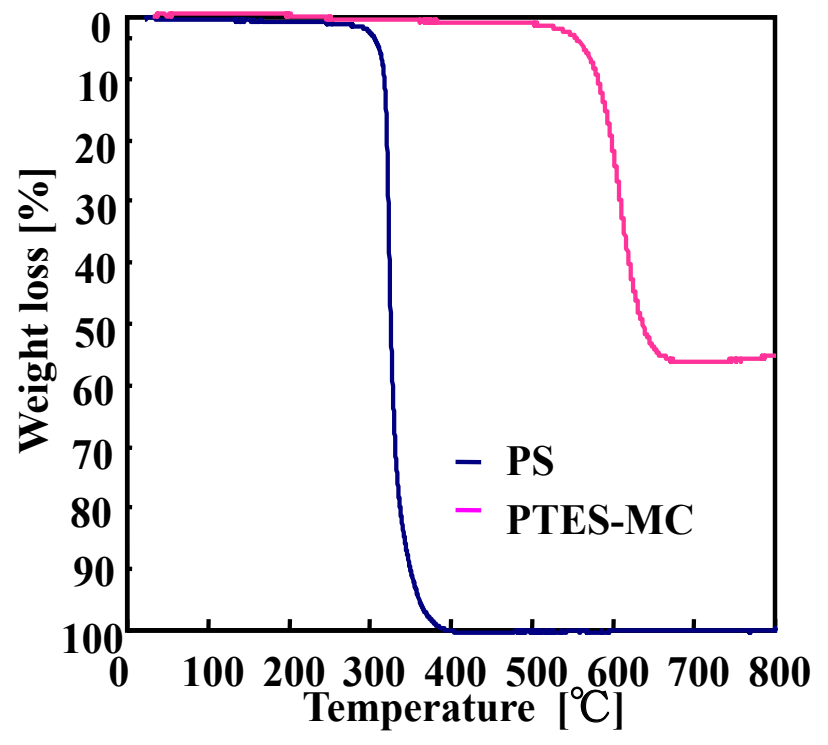

Figure 5. TG Curves of PTES-MC and PS
PTES-MCs and the amount of PTES sol dripped into the ammoniacatalyst solution. Accordingly, by controlling those conditions, it was possible to control MC particle size. Change in diameter of MCs with raw-material concentration (PTES sol) is plotted in Figure 6. The dropping amount at each PTES-sol concentration was fixed at $1.2 \times 10^{-2}$ g. Although diameter of $300 \mu \mathrm{m}$ was obtained at PTES concentration of $100 \mathrm{wt} \%$, when PTES-sol concentration was reduced in the order $80 \% \rightarrow 60 \% \rightarrow 40 \% \rightarrow 20 \% \rightarrow 10 \%$, MC particle diameter was reduced in the order $150 \mu \mathrm{m} \rightarrow 120 \mu \mathrm{m} \rightarrow 50 \mu \mathrm{m} \rightarrow 20 \mu \mathrm{m} \rightarrow 5 \mu \mathrm{m}$.

Change in mean particle diameter with PTES-sol concentration with PTES dropping weight as a parameter is plotted in Figure 7. When dropping weight was reduced from $1.2 \times 10^{-2} \mathrm{~g}$ to $3.0 \times 10^{-3} \mathrm{~g}$, mean particle diameter was significantly decreased. In other words, nanometer-sized (i.e., sub-micron level) particles were obtained. At PTES concentration of $60 \%$, particle diameter dramatically was reduced, namely, from 120 to $5 \mu \mathrm{m}$. At PTES concentrations of $40 \%$ and $20 \%$, particle diameters were 2 and $1 \mu \mathrm{m}$, respectively. At the PTES concentration of $10 \%$, nanometer-sized MCs (i.e., diameter of about several hundred nanometers) were obtained. When the PTES sol was dropped into the base catalyst $\left(\mathrm{NH}_{4} \mathrm{OH}\right)$, particulate MCs were formed immediately. It is presumed that since the PTES sol is insoluble in an aqueous solution, the sol-gel reaction proceeds on the surface in a three-dimensional direction as soon as the droplet of the sol contacts the base-catalyst surface. And since the size of the droplet is determined by the volume of sol dropped, the sol-droplet volume has a significant effect on the size of the MCs formed.

Observation images (taken by SEM) of micrometer-sized and nanometer-size MCs are shown in Figure 8. It is clear from these SEM images that both the micrometer-sized and nanometer-sized MCs are spherical. MCs and NCs (nanocapsules) show significantly different heat resistance. Change in the surface morphology of MCs and NCs heat-treated at 180, 200, and $220^{\circ} \mathrm{C}$ are shown in Figure 9. In this temperature range, the MCs show no change in morphology; in contrast, the NCs start to melt and fuse together when heated above $200^{\circ} \mathrm{C}$. It is considered that the difference in the two types of capsules in terms of thermal energies is derived from the difference in specific surface areas of the particles. Such reduction of the melting point by this so-called "nano-sizing" is a phenomenon also known to occur in the case of other particles (e.g., fine metal particles) [18].

In the manner explained above, by controlling PTES-sol concentration and volume of PTES-sol dropped into the base catalyst, it is possible to obtain MCs with size ranging from the sub-micron level to around $300 \mu \mathrm{m}$ and to control particle diameter by controlling the synthesis conditions during fabrication of MCs. It will be possible to more precisely control particle size by centrifugation of the prepared particles. That matter will be reported in a future paper.

\section{Synthesis and properties of microencapsulated latent pigment}

As for synthesis of PTES-MCs, latent pigment dissolved in solvent (dioxane) was added to sol solution (formed by polymerization of PTES by acid catalyst), and when this mixed solution was dropped into a basic catalyst solvent, a microencapsulated latent pigment was formed. The chemical structures of the latent pigments used are shown in Figure 10. Three primary-color pigments, namely, quinacridoneBOC (for magenta), indigo-BOC (for cyan), and Pigment Yellow 93$\mathrm{BOC}$ (PY-BOC) (for yellow), were selected for the latent pigmentation. In the three kinds of latent pigments, the hydrogen of the $\mathrm{N}-\mathrm{H}$ group in each organic pigment molecule was substituted with a t-BOC $\left(\mathrm{COOC}\left(\mathrm{CH}_{3}\right)_{3}\right)$ group, and all three kinds were soluble in an organic 


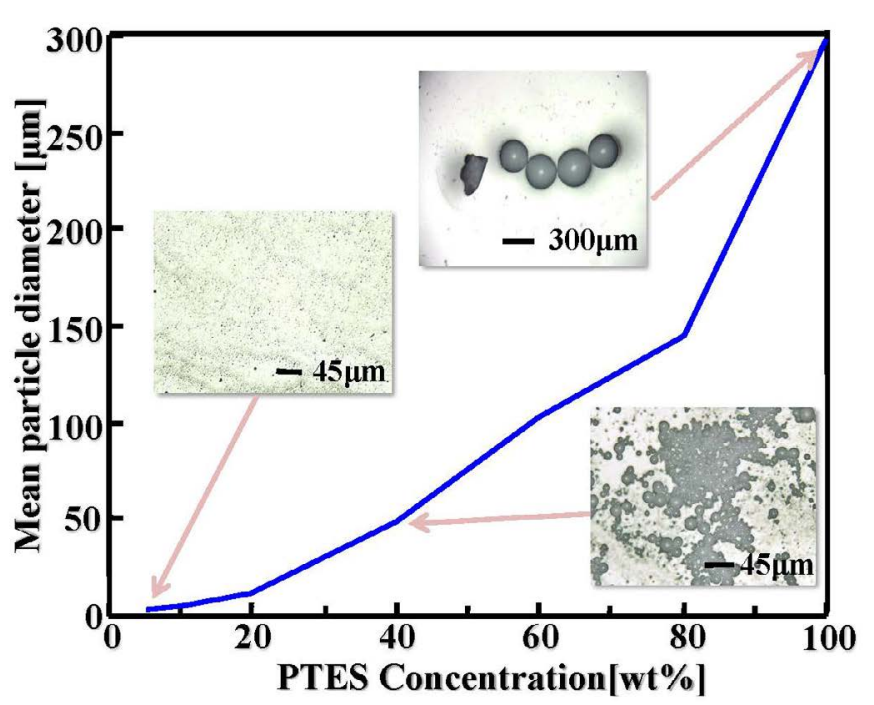

Figure 6. Relationship between PTES concentration and mean diameter of MC

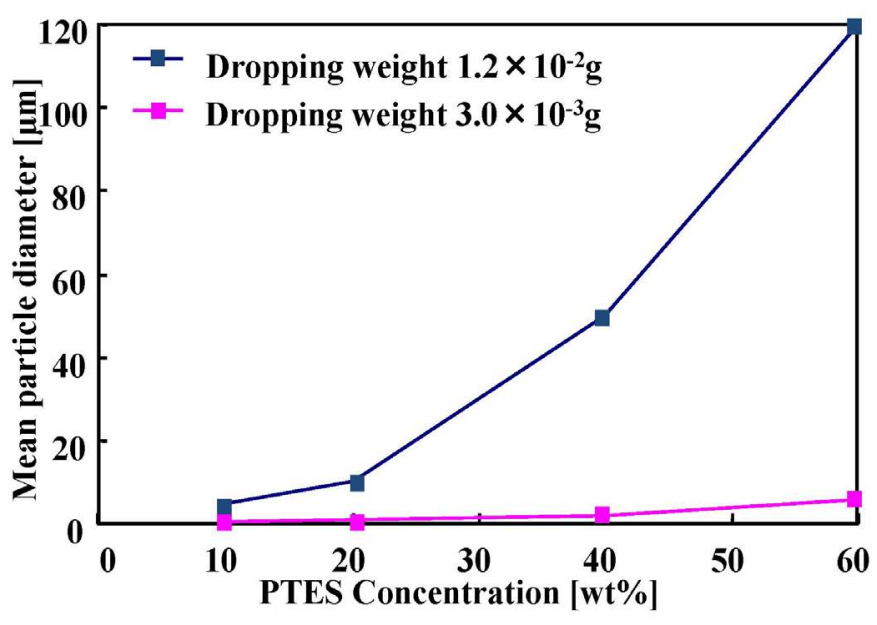

Figure 7. Relationship between PTES dropping weight and mean diameter of MC

\section{Micro-size capsule}

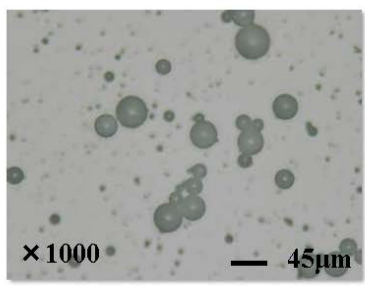

10 $40 \mu \mathrm{m}$

\section{Nano-size capsule}

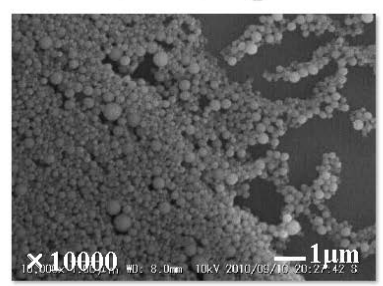

$100 \sim 500 \mathrm{~nm}$
Figure 8. SEM photographs of micro-size and nano-size capsules

solvent such as 1,4-dioxane. As for the MCs containing the latent pigments, under heat treatment in the range of 160 to $180^{\circ} \mathrm{C}$, the $\mathrm{t}-\mathrm{BOC}$ group of the latent pigment was eliminated, and when isobutylene gas $\left(\mathrm{H}_{2} \mathrm{C}=\mathrm{C}\left(\mathrm{CH}_{3}\right)_{2}\right)$ and $\mathrm{CO}_{2}$, which were generated by decomposition of $\mathrm{t}$-BOC, were removed from the system, the pigment was formed inside the MCs.

Surface states of the microencapsulated latent pigments before and after heat treatment are shown in Figure 11. When the MCs containing the latent pigments were heat treated at $160^{\circ} \mathrm{C}$, spherical MCs colored with the three primary-color materials, namely, red-purple (quinacridone, Qn), blue (indigo), and yellow (PY93), were obtained. It is clear that colors of the MCs differ considerably before and after the heat treatment and that the pigment is formed inside the MCs due to the heat treatment. Moreover, the MC surface after heat treatment was smooth, and porosity due to gas generated when the latent pigment transforms to the pigment was not observed on the surface of the MCs. By varying the mixing ratio of the three primary-color latent pigments of this color material, it is possible to develop MCs with various colors. Multi-colored MCs prepared by mixing the three primary-color latent pigments are shown in Figure 12. By systematically varying the mixing ratios of PY93/Qn, indigo/Qn, and PY93/indigo from 9: 1 to 1: 9, it was possible to produce inorganic MCs with various colors, including red, blue, and green (namely, the three primary colors of light). In addition, it was possible to prepare nanoencapsulated organic pigment by controlling the particle diameter during preparation of the inorganic MCs.

\section{Light-resistancetestofinorganic-material-microencapsulated} organic pigment

Light resistance is one factor that becomes important when coloring materials are applied in optical materials. It is a particularly important property in cases such as when continuity of coloration (e.g., storage stability of a pigment) must be necessary. In light of that requirement, a light-resistance test on the prepared inorganic-microencapsulated organic pigment was performed. The result of that test on microcapsulated PTES-Qn pigment is shown in Figure 13 in comparison with PS encapsulated Acid Red (organic polymer encapsulated dye). The relationship between UV exposure time and change in maximum absorption wavelength for each sample is shown in Figure 13.

A sample in which inorganic MCs containing Qn (PTES-MCs) were coated as a film on a glass substrate was prepared and tested. As a comparison target, polystyrene MCs (PS-MCs) containing a rhodamine dye called Acid Red 52 (sulfophorhodamine B sulphonylchloride) were also prepared and tested. Acid Red 52 was chosen because it is a typical magenta-coloring material and has an absorption peak at 560 to 550 $\mathrm{nm}$ in almost the same manner as that of $\mathrm{Qn}$.

As for the test method, the samples were irradiated with ultraviolet rays (with wavelength of $365 \mathrm{~nm}$ and intensity of $614 \mu \mathrm{W} / \mathrm{cm}^{2}$ ), and the change with time of the transmittance of the films at $550 \mathrm{~nm}$ was recorded. One sample was irradiated for 24 hours, and the temporal change of the UV/VIS transmittance spectrum for the maximum absorption wavelength (Qn: $550 \mathrm{~nm}$; Acid Red: $560 \mathrm{~nm}$ ) of the pigment was calculated under the assumption that the transmittance at time zero was $100 \%$.

Since the film was made by using MCs, transmittance varied according to location; despite that variation, the transmittance of the inorganic-material-microencapsulated Qn pigment varied only slightly.

In contrast, as for the PS MCs containing Acid Red, transmittance decreased significantly with passage of time, and after 24 hours, it decreased by about $30 \%$. This result can be explained by the fact that Acid Red (which is an organic dye) and PS (which is an organic resin) are decomposed by ultraviolet rays. It is clear from the above results that the Qn-containing inorganic MCs developed in this study show significantly improved light resistance compared to the PS MCs (organic resin) using conventional organic dye. Moreover, as for the MCs containing yellow (PY93) and cyan (Indigo) pigments, according to accelerated UV-irradiation tests, decrease in transmittance was not 


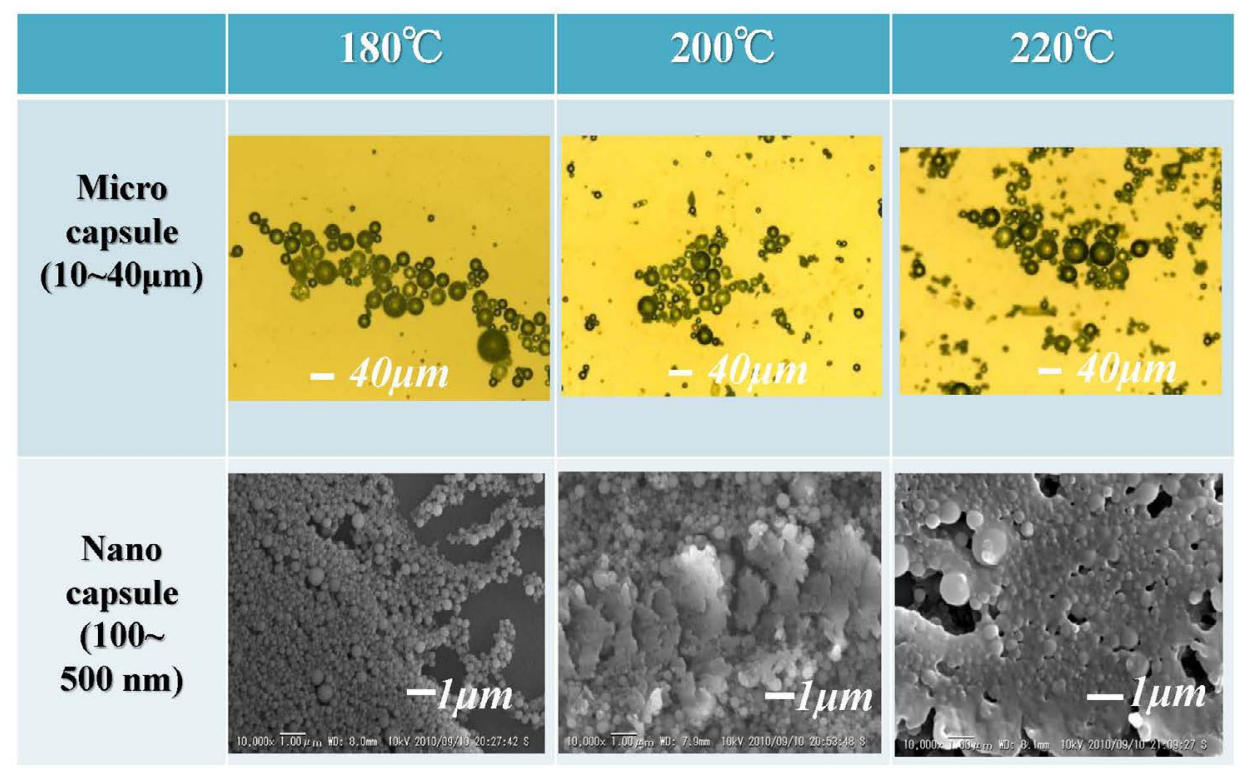

Figure 9. Surface morphology of micro- and nano- capsules after heat treatment

\section{Pigment Yellow 93-BOC}
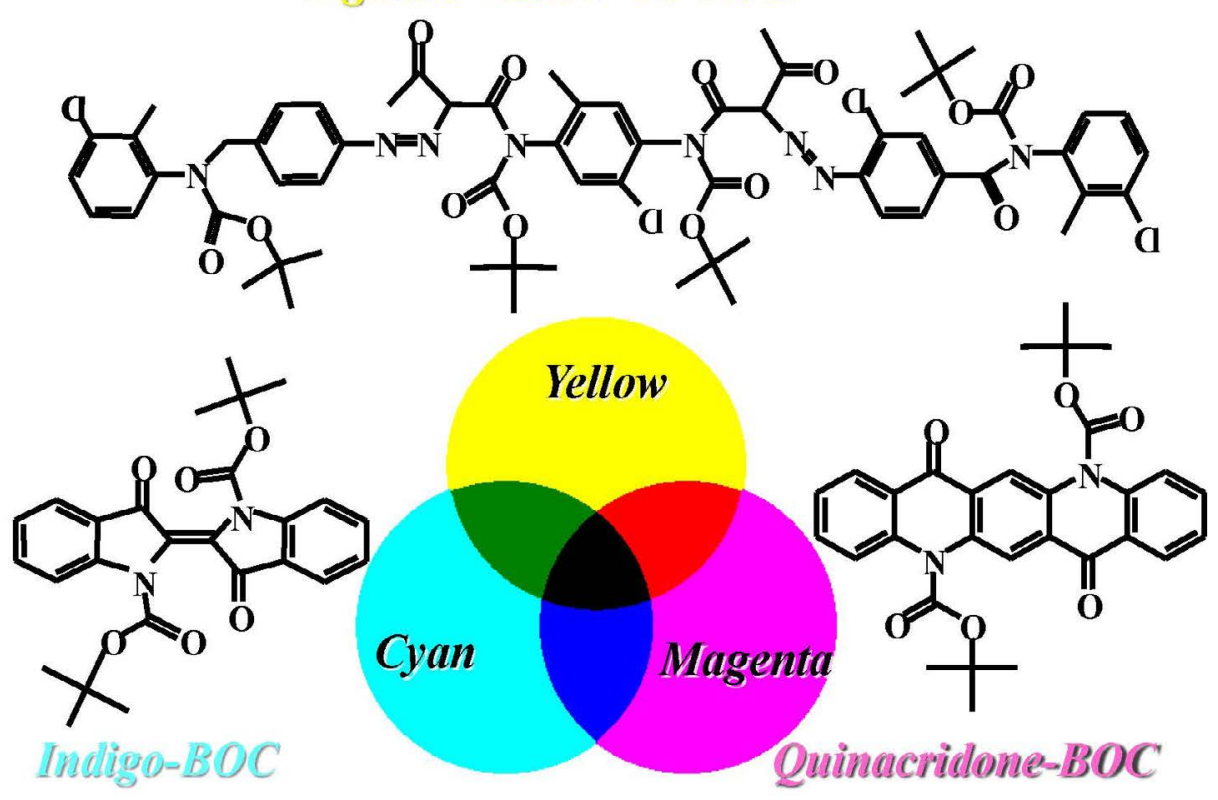

Figure 10. Chemical structure of three primary colors of latent pigments

observed, so it is concluded that the light resistance of the developed microencapsulated pigment is extremely high.

General organic pigments are insoluble in solvents, so fabricating a homogenous system with macromolecules used for microencapsulation is difficult; moreover, it is not easy to introduce them into a sol-gel film because organic pigment has an aromatic ring in a molecule and shows hydrophobic properties, compatibility to sol-gel solution synthesized in a polar solvent is not good.

A latent pigment is easy to introduce into the sol-gel derived inorganic MCs because it is soluble in a solvent, and $\mathrm{Ph}-\mathrm{SiO}_{2}$ (used in this study) has a phenyl group, so its compatibility with an organic pigment is high. Moreover, converting the latent pigment inside MCs to organic pigment is easy by heating at about $160^{\circ} \mathrm{C}$. Since the shape of the organic-pigment-containing inorganic MCs made in this study is spherical, it is easy to pack them uniformly in a matrix and prepare dispersed solutions. The organic pigment, with high thermal and light resistance, is embedded in an inorganic network; consequently, the durability of the fabricated multicolored inorganic MCs is high. As a result, it is considered possible to develop it in a wide range of applications such as pigments for inkjets and toners and pigments for decoration.

Fluorescence-emission characteristics of micro- and nanoencapsulated Qn-BOC latent pigment

The latent pigment Qn-BOC showed strong fluorescence. Fluorescence emission spectrum of Qn-BOC in ethanol solution and 


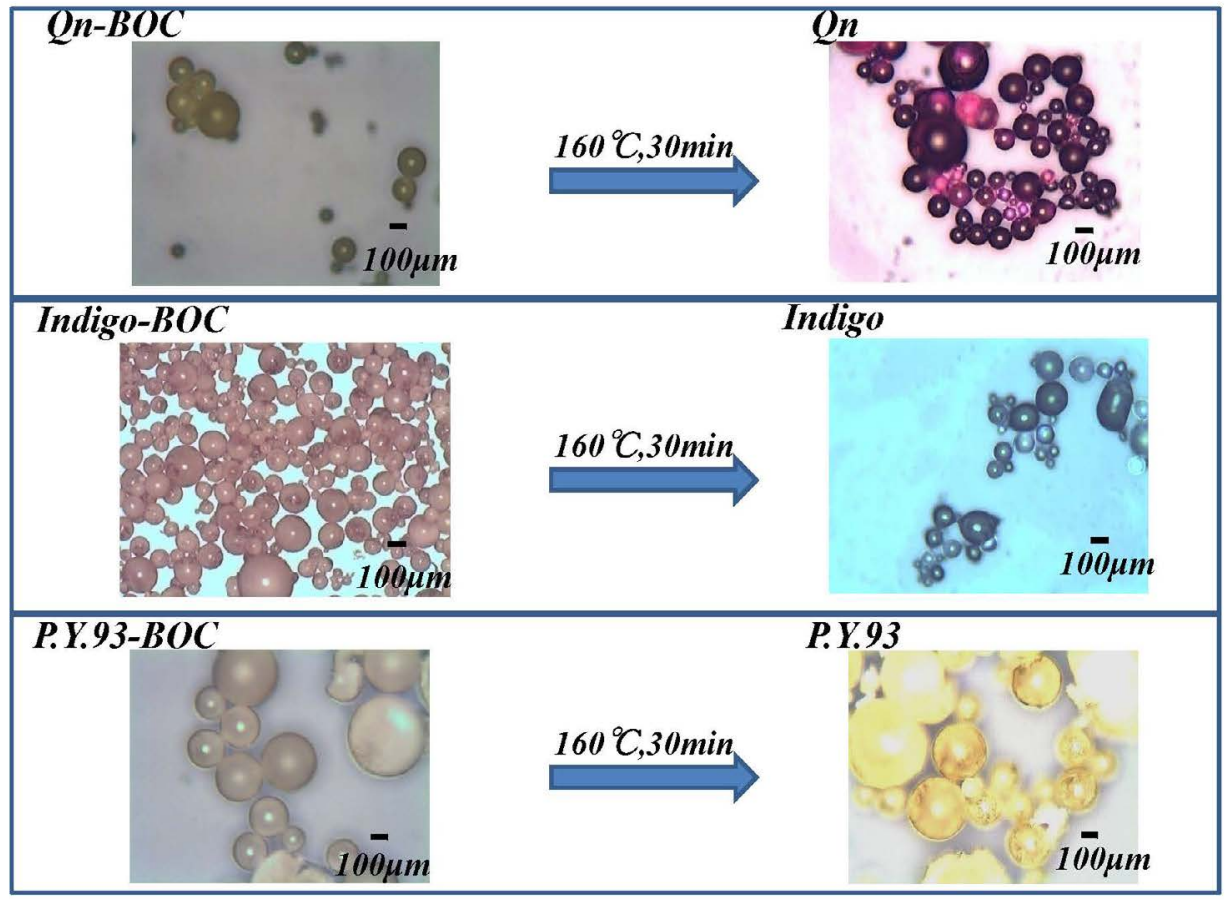

Figure 11. Color change of micro capsules containing three primary colors of latent pigments by heat treatment

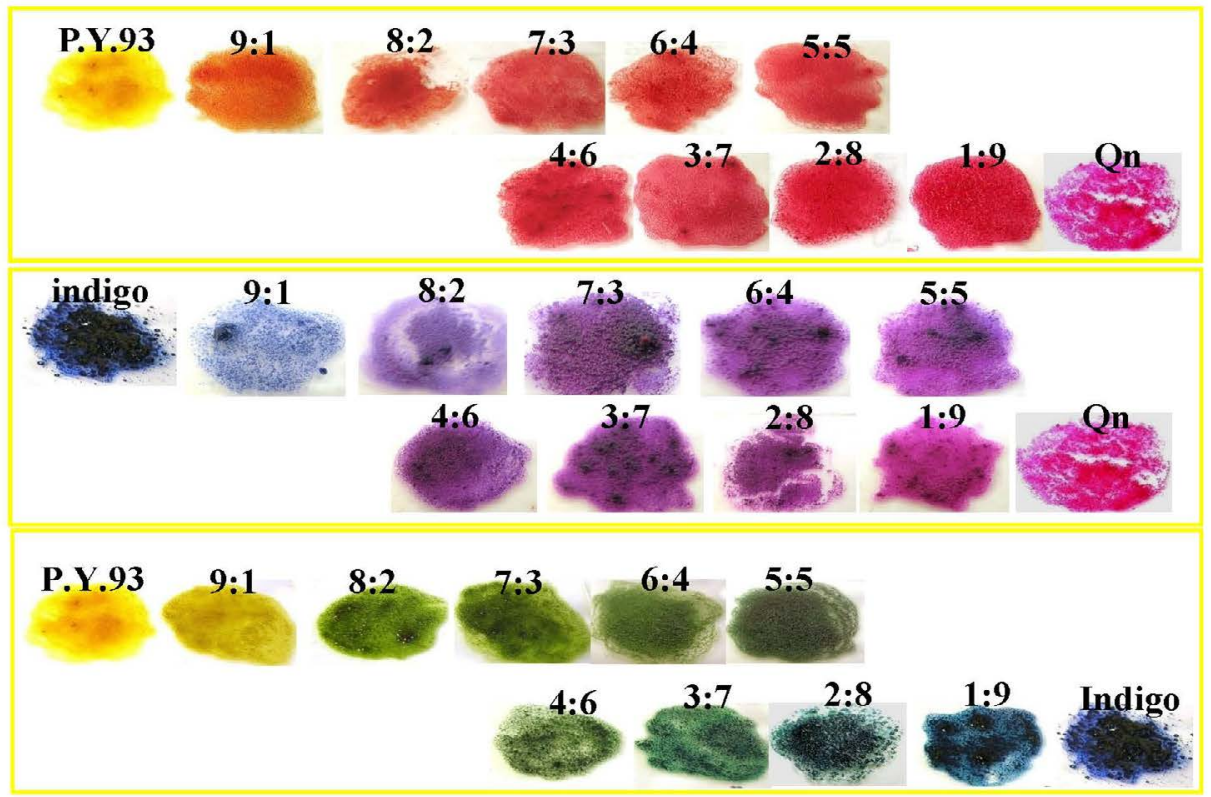

Figure 12. Multi-colored micro-capsules prepared by mixing three primary colors of latent pigments

emission state of Qn-BOC nanocapsules are shown in Figure 14. Although Qn-BOC shows absorption peak at $450 \mathrm{~nm}$, when UV light with excitation wavelength of $284 \mathrm{~nm}$ (Qn-BOC has a strong absorption with molar absorption coefficient of $\log \varepsilon=5$ at $284 \mathrm{~nm}$ ) was irradiated onto the solution, an emission spectrum with a peak at $510 \mathrm{~nm}$ was obtained [Figure 14(a)]. Both the Qn-BOC-containing inorganic MCs and nanocapsules showed a stronger fluorescent property. The state (yellow-green) of fluorescence emission when UV light was irradiated onto the Qn-BOC nanocapsules is shown in Figure 14(b). By using the dispersed solution of nanocapsules with an inkjet, it was possible to print a character pattern. When the character pattern was irradiated with UV light, it emerged as fluorescent emission, and it was possible to define various character styles [Figure 14(c)]. Since complex-shaped patterns can be printed by inkjet, it is conceivable that the nanoparticles can be applied to fluorescent labeling, for example.

\section{Conclusion}

Three basic primary-color latent pigments (Qn-BOC, Indigo-BOC, and $\mathrm{PY}-\mathrm{BOC}$ ) as coloring materials and $\mathrm{Ph}-\mathrm{SiO}_{2}$ (synthesized by the sol-gel method) were used to synthesize organic-pigment-containing inorganic micro- and nanocapsules. By controlling the sol-gel reaction between acidic catalyst and basic catalyst, it was possible to prepare 


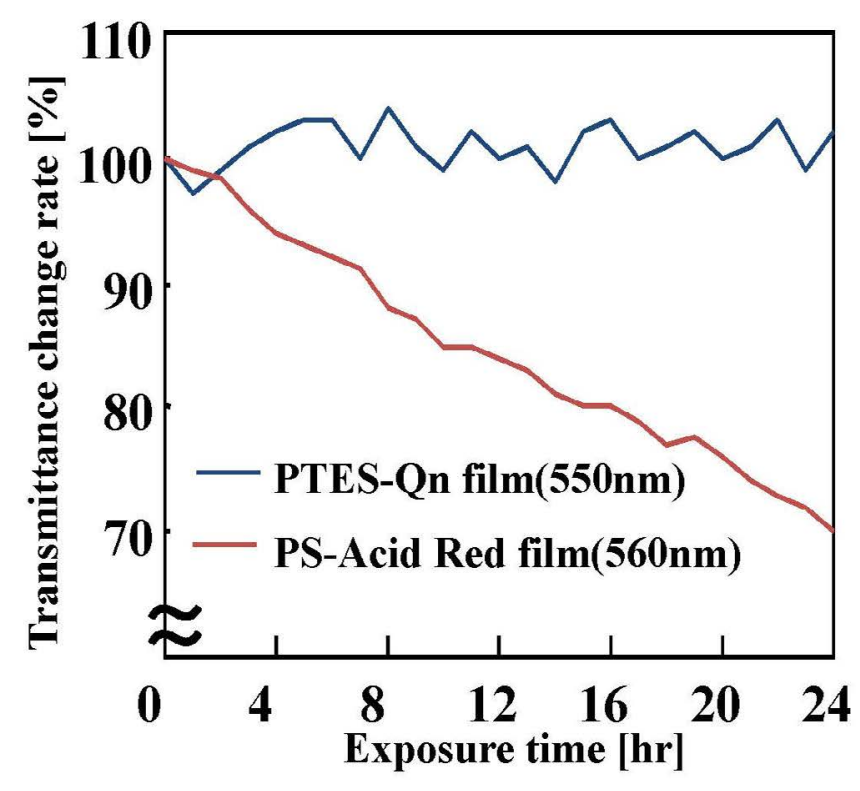

Figure 13. Durability against ultraviolet of micro-encapsulated Qn pigment

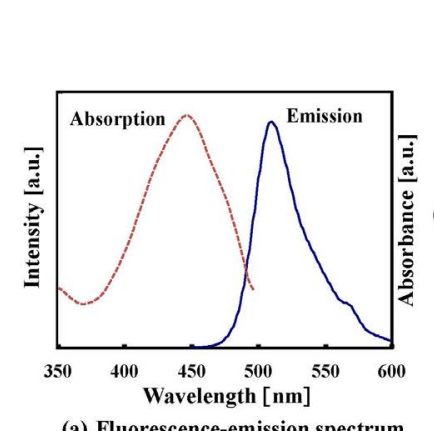

(a) Fluorescence-emission spectrum in ethanol

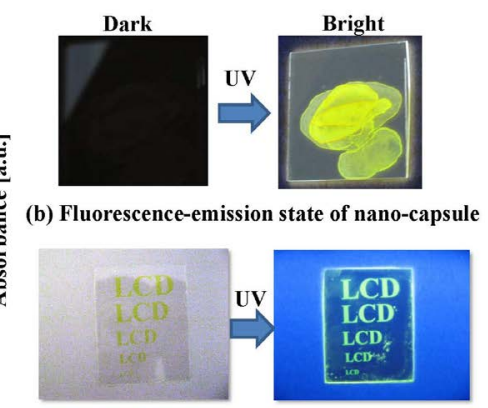

(c) Inkjet pattern of nano-capsule
Figure14. Fluorescence emission spectrum of Qn-BOC and emission state of nanoencapsulated Qn-BOC and inkjet patterned film on glass

spherical inorganic capsules. Since the latent pigment was soluble in an organic solvent, it was easily taken into inorganic capsules and converted into an organic pigment by heat treatment. The organic pigment was enclosed in the inorganic capsules, and colored MCs with excellent heat resistance, light resistance, and coloring property were obtained. By changing the composition ratio of the three basic primarycolor latent pigments, it was possible to create inorganic capsules with various colors. Inorganic micro- and nanocapsules of Qn-BOC showed strong fluorescence-emission characteristics, and various complicated fluorescent-pattern films were fabricated by the inkjet method using a dispersed solution containing the capsules.

\section{References}

1. Omodani M (2004) Current Technologies and Application for Electric Paper, CMC.

2. Bouchard A, Suzuki K, Yamada H (2004) High-resolution microencapsulated electrophoretic display on silicon, SID 04 Digest 651-653.

3. Duthaler G, Au J, Davis M, Gates H, Hone B, et al. (2002) Active-matrix color displays using electrophoretic ink and color filters, SID 02 Digest, 1374-1377.

4. Sawatani N, Fukuda M, Taguchi Y, Tanaka M (2005) Composite polymer particles with a gradated resin composition by suspension polymerization, J. Applied Polymer Science 97: 682-690.

5. Slobodian P, Pavlinek V, Lengalova A, Saha S (2009) Polystyrene/multi-wall carbon nanotube composites prepared by suspension polymerization and their electrophological behavior, Current Applied Physics 9: 184-188.

6. Kutsuwada M, Taguchi Y, Tanaka M (2015) Preparation of composite particles made of biodegradable polymer and a few additives with phase separation followed by dryingin-ligand, J. Encapsulation and adsorption Science 5, 11-20.

7. Tsutsumi T (2006) Encapsulated pigment for ink jet application, J. Imaging Society of Japan 45, 29-34.

8. Shibata S, Yamane M, Kamada K, Ohta K, Sasaki K, et al. (1997) Laser emission from dye-doped organic-inorganic particles of microcavity structure, J. Sol-Gel Science and Technology 8, 959-964.

9. Verma NK, Crosbie-Staunton K, Satti A, Gallagher S, Ryan KB, et al. (2013) Magnetic core-shell nanoparticles for drug delivery by nebulization. J Nanobiotechnology 11 : 1. [Crossref]

10. Deng J, Peng Y, Hen C, Long X, Chan ASC (2003) Magnetic and conducting Fe3O4polypyrrole nanoparticles with core-shell structure, Polymer International 52, 11821187.

11. Kimura I, Ikarashi S, Saito N, Tanaka M (1997) Microencapsulation of thermalsensitive pigment with interfacial reaction in multiple dispersion, Advanced Powder Technology 8, 1-13.

12. Ding Y, Chen Q, Qian H, Chen Y, Wu W, et al. (2009) Gold encapsulated chitosan-poly (acrylic acid) hybrid hollow nanosphere, Macromolecular Bioscience 9, 1272-1280.

13. Matsumoto F, Nishio K, Miyasaka T, Masuda H (2004) Ideally ordered, high-density patterning of DNA on Au disk array fabricated using anodic porous alumina, Jpn. J. Appl. Phys. 43, L640.

14. Organic Pigments Handbook (2006) Color Office.

15. Hao Z, Iqbal A (1997) Some aspects of organic pigments, Chemical Society Reviews 26, 203-213.

16. Zambounis Z, Hao H, Iqbal A (1997) Latent pigments activated by heat, Nature 388, 131-132.

17. a) Ohishi T, Sugawara S (2014) Preparation and optical patterning of organic-inorganic hybrid color-filter films using latent pigments by utilizing photo-acid-generator and microwave irradiation, Materials Sciences and Application 5, 1079-1090.

18. b) Ohishi T, Ishitsuka H (2011) Preparation and properties of sol-gel thin film containing quinacridone latent pigment by using laser irradiation, Materials Science and Engineering 18, 032010.

19. Buffat PH, Borrel JP (1976) Size effect on the melting temperature of gold particles, Physical Review A 13, 2287-2298.

Copyright: (C2017 Ohishi T. This is an open-access article distributed under the terms of the Creative Commons Attribution License, which permits unrestricted use, distribution, and reproduction in any medium, provided the original author and source are credited. 